\title{
When is biopsy-proven TIN not simply TIN? Questions
}

\author{
Nicholas Ware\& Neil J. Sebire W. K. Chong\& Rajesh Krishnan, Stephen D. Marks
}

A 7.5-year-old boy presented to a community clinic looking generally unwell. He was referred to our hospital and found to have end-stage kidney disease.

He was born at $40+3$ weeks gestation to non- consanguineous parents by normal vaginal delivery following spontaneous onset of labour after a maternal antepartum haemorrhage. His antenatal ultrasounds were normal at $12,20,22,30,32$ and 34 weeks. He was born in good condition, with a birth weight of $4 \mathrm{~kg}$, and discharged on day 2 of life.

From the time he was discharged home his mother was concerned about nystagmus, and at 8 weeks he was referred to the ophthalmologist as he was not fixing and following. He was reviewed by both a developmental paediatrician and an ophthalmologist on a monthly basis and was diagnosed with hypermetropia requiring glasses at 9 months of age. He had a cranial magnetic resonance imaging (MRI) scan at 11 months of age which was reported to be normal (Fig. 1).

He was noted to have global developmental delay. Blood tests were taken at the age of 2 years as part of a genetics

work-up, and these showed normal renal function (plasma creatinine $36 \mu \mathrm{mol} / \mathrm{L}$, urea $3.5 \mathrm{mmol} / \mathrm{L}$, sodium 141 $\mathrm{mmol} / \mathrm{L}$, potassium $4.5 \mathrm{mmol} / \mathrm{L}$ and haemoglobin $121 \mathrm{~g} / \mathrm{L}$ ). He did not walk independently until the age of 4.5 years and was diagnosed with an autistic spectrum disorder at 7 years of age (only had four words at 9 years of age).

He was diagnosed with pharyngitis and given oral antibi- otics by his general practitioner at the age of 7.5 years. A few weeks later he presented to a community clinic looking gen- erally unwell and was noted to be pale with a flow murmur. He was referred to hospital, and on admission his weight and height were $22.3 \mathrm{~kg}$ ( $>9$ th centile) and $117.1 \mathrm{~cm}$ (<9th centile), respectively, and he was hypertensive with systolic blood pressure of $155 \mathrm{mmHg}$. His urine protein:creatinine ratio was $124 \mathrm{mg} / \mathrm{mmol}$, and his blood tests revealed a plasma

creatinine level of $1262 \mu \mathrm{mol} / \mathrm{L}$, urea $58 \mathrm{mmol} / \mathrm{L}$, sodium $142 \mathrm{mmol} / \mathrm{L}$, potassium $4.2 \mathrm{mmol} / \mathrm{L}$, corrected calcium $1.56 \mathrm{mmol} / \mathrm{L}$, phosphate $2.52 \mathrm{mmol} / \mathrm{L}$, bicarbonate $6 \mathrm{mmol} / \mathrm{L}$ and haemoglobin $55 \mathrm{~g} / \mathrm{L}$.

Following admission and stabilisation, he was referred to a tertiary nephrology unit due to his deranged renal function, persistent hypocalcaemia and metabolic acidosis. A renal ul- trasound scan showed loss of corticomedullary differentiation and increased echogenicity, with his right kidney measuring $8.2 \mathrm{~cm}$ and left kidney measuring $9.2 \mathrm{~cm}$ (5th and 50th centiles for age are $7.4 \mathrm{~cm}$ and $8.8 \mathrm{~cm}$, respectively).

He then underwent a percutaneous renal biopsy under gen- eral anaesthetic, which contained 41 glomerular profiles of which five $(12 \%)$ were globally sclerosed. Two showed periglomerular fibrosis, and a number of the others showed mild ischaemic shrinkage. There was no mesangial thicken- ing, hypercellularity or segmental lesions. Glomerular base- ment membranes showed mild ischaemic shrinking on silver

stain only, with negative Congo red staining for amyloid. There was focal tubular atrophy and diffuse interstitial oedema with a dense diffuse interstitial inflammatory infiltrate of lym- phocytes. In several foci lymphocytes were seen to infiltrate tubules and tubular rupture was noted. There were no specific glomerular deposits as revealed by immunocytochemistry (IgG, IgA, IgM or C3). Three sclerosed and one viable glo- merulus were visible upon electron microscopy examination, all of which showed some ischaemic shrinkage and foot process fusion but no deposits. The conclusion drawn from the biopsy results was acute tubulointerstitial nephritis (TIN), with the presence of sclerosis and atrophy consistent with a degree of chronicity (Fig. 2).

The patient was commenced on corticosteroid therapy which he remained on for 4 months. Haemodialysis was started at presentation, and he has required this since. On referral to our quaternary paediatric nephrology service for transplant work-up at the age of 9 years, his history was reviewed and it was felt that he exhibited a 
number of features of Joubert syndrome (JS). His previous MRI scan was reviewed again and confirmed the pathognomic sign of JS. The results of his genetics work-up were sent, and further review also confirmed the diagnosis of JS.

\section{Questions}

1. What is the pathognomic sign of JS?

2. What are the clinical features of JS?

3. What are the renal manifestations typically seen in JS

including the radiological and histological findings?

4. What features of this boy's biopsy was unusual for JS? 